\title{
Research Article \\ Some Geometric Properties of Lacunary Sequence Spaces Related to Fixed Point Property
}

\author{
Chirasak Mongkolkeha and Poom Kumam
}

Department of Mathematics, Faculty of Science, King Mongkut's University of Technology Thonburi (KMUTT), Bangmod, Thrungkru, Bangkok 10140, Thailand

Correspondence should be addressed to Poom Kumam, poom.kum@kmutt.ac.th

Received 18 June 2011; Accepted 2 November 2011

Academic Editor: Ondřej Došlý

Copyright (C 2011 C. Mongkolkeha and P. Kumam. This is an open access article distributed under the Creative Commons Attribution License, which permits unrestricted use, distribution, and reproduction in any medium, provided the original work is properly cited.

The main purpose of this paper is considering the lacunary sequence spaces defined by Karakaya (2007), by proving the property $(\beta)$ and Uniform Opial property.

\section{Introduction}

Let $(X,\|\cdot\|)$ be a real Banach space and let $B(X)$ (resp., $S(X)$ ) be a closed unit ball (resp., the unit sphere) of $X$. For any subset $A$ of $X$, we denote by $\operatorname{conv}(A)$ the convex hull of $A$. The Banach space $X$ is uniformly convex (UC), if for each $\varepsilon>0$ there exists $\delta>0$ such that for $x, y \in S(X)$ the inequality $\|x-y\|>\varepsilon$ implies $\|(x+y) / 2\|<1-\delta$ (see [1]). A Banach space $X$ has the property $(\beta)$ if for each $\varepsilon>0$ there exists $\delta>0$ such that $1<\|x\|<$ $1+\delta$ implies $\alpha(\operatorname{conv}(B(X) \cup\{x\}) \backslash B(X))<\varepsilon$, where $\alpha(A)$ denotes the Kuratowski measure noncompactness of a subset $A$ of $X$ defined as the infimum of all $\varepsilon>0$ such that $A$ can be covered by a finite union of sets of diameter less than $\varepsilon$. The following characterization of the property $(\beta)$ is very useful (see [2]): A Banach space $X$ has the property $(\beta)$ if and only if for each $\varepsilon>0$ there exists $\delta>0$ such that for each element $x \in B(X)$ and each sequence $\left(x_{n}\right)$ in $B(X)$ with $\operatorname{sep}\left(x_{n}\right) \geq \varepsilon$ there is an index $k$ for which $\left\|\left(x+x_{k}\right) / 2\right\|<1-\delta$ where $\operatorname{sep}\left(x_{n}\right)=\inf \left\{\left\|x_{n}-x_{m}\right\|: n \neq m\right\}>\varepsilon$. A Banach space $X$ is nearly uniformly convex (NUC) if for each $\varepsilon>0$ and every sequence $\left(x_{n}\right)$ in $B(X)$ with $\operatorname{sep}\left(x_{n}\right) \geq \varepsilon$, there exists $\delta \in(0,1)$ such that $\operatorname{conv}\left(x_{n}\right) \cap(1-\delta) B(X) \neq \emptyset$. Define for any $x \notin B(X)$ the drop $D(x, B(X))$ determined by $x$ by $D(x, B(X))=\operatorname{conv}(B(X) \cup\{x\})$. A Banach space $X$ has the drop property (write $(D))$ if for every closed set $C$ disjoint with $B(X)$ there exists an element $x \in C$ such that $D(x, B(X)) \cap C=\{x\}$. A point $x \in S(X)$ is an $H$-point of $B(X)$ if for any sequence $\left(x_{n}\right)$ in $X$ such that $\left\|x_{n}\right\| \rightarrow 1$ as $n \rightarrow \infty$, the week convergence of $\left(x_{n}\right)$ to $x$ implies that $\left\|x_{n}-x\right\| \rightarrow 0$ as 
$n \rightarrow \infty$. If every point in $S(X)$ is an $H$-point of $B(X)$, then $X$ is said to have the property $(H)$. A Banach space is said to have the uniform Kadec-Klee property (abbreviated as (UKK)) if for every $\varepsilon>0$ there exists $\delta>0$ such that for every sequence $\left(x_{n}\right)$ in $S(X)$ with $\operatorname{sep}\left(x_{n}\right) \geq \varepsilon$ and $x_{n} \stackrel{w}{\longrightarrow} x$ as $n \rightarrow \infty$, we have $\|x\|<1-\delta$. Every (UKK) Banach space has $H$-property (see [3]). The following implications are true in any Banach spaces,

$$
\begin{gathered}
(D) \Longrightarrow(R f x) \\
\text { 介 } \\
(\mathrm{UC}) \Longrightarrow \operatorname{property}(\beta) \Longrightarrow(\mathrm{NUC}) \Longrightarrow(\mathrm{UKK}) \Longrightarrow \operatorname{property}(H),
\end{gathered}
$$

where $(R f x)$ denotes the property of reflexivity (see [3-6]). A Banach space $X$ is said to have the Opial property (see [7]) if every sequence $\left(x_{n}\right)$ weakly convergent to $x_{0}$ satisfies

$$
\lim _{n \rightarrow \infty} \inf \left\|x_{n}-x_{0}\right\| \leq \lim _{n \rightarrow \infty} \inf \left\|x_{n}-x\right\|
$$

for every $x \in X$. Opial proved in [7] that the sequence space $l_{p}(1<p<\infty)$ have this property but $L_{p}[0, \pi](p \neq 2,1<p<\infty)$ do not have it. A Banach space $X$ is said to have the uniform Opial property (see [8]), if for each $\varepsilon>0$ there exists $\tau>0$ such that for any weakly null sequence $\left(x_{n}\right)$ in $S(X)$ and $x \in X$ with $\|x\| \geq \varepsilon$ there holds

$$
1+\tau \leq \lim _{n \rightarrow \infty} \inf \left\|x_{n}+x\right\|
$$

For example, the space in $[9,10]$ has the uniform Opial property. The Opial property is important because Banach spaces with this property have the weak fixed point property (see $[11])$ and the geometric property involving fixed point theory can be found, for example, in $[9,12-14]$.

by

For a bounded subset $A \subset X$, the set measure of noncompactness was defined in [15]

$$
\alpha(A)=\inf \{\varepsilon>0: A \text { can be covered by finitely many sets of diameter } \leq \varepsilon\} .
$$

The ball measure of noncompactness was defined in $[16,17]$ by

$$
\beta(A)=\inf \{\varepsilon>0: A \text { can be covered by finitely many balls of diameter } \leq \varepsilon\} .
$$

The functions $\alpha$ and $\beta$ are called the Kuratowski measure of noncompactness and the Hausdorff measure of noncompactness in $X$, respectively. We can associate these functions with the notions of the set-contraction and ball contraction (see [18]). These notions are very useful tools to study nonlinear operator propblems (see $[8,18]$ ). For each $\varepsilon>0$ define that $\Delta(\varepsilon)=$ $\inf \{1-\inf [\|x\|: x \in A]: A$ is closed convex subset of $B(X)$ with $\beta(A) \geq \varepsilon\}$. The function $\Delta$ is called the modulus of noncompact convexity (see [16]). A Banach space $X$ is said to have property $(L)$ if $\lim \varepsilon_{\rightarrow 1^{-}} \Delta(\varepsilon)=1$. It has been proved in [8] that property $(L)$ is a useful tool in 
the fixed point theory and that a Banach space $X$ has property $(L)$ if and only if it is reflexive and has the uniform Opial property.

For a real vector space $X$, a function $\rho: X \rightarrow[0, \infty]$ is called a modular if it satisfies the following conditions:

(i) $\rho(x)=0$ if and only if $x=0$;

(ii) $\rho(\alpha x)=\rho(x)$ for all scalar $\alpha$ with $|\alpha|=1$;

(iii) $\rho(\alpha x+\beta y) \leq \rho(x)+\rho(y)$, for all $x, y \in X$ and all $\alpha, \beta \geq 0$ with $\alpha+\beta=1$;

the modular $\rho$ is called convex if

(iv) $\rho(\alpha x+\beta y) \leq \alpha \rho(x)+\beta \rho(y)$, for all $x, y \in X$ and all $\alpha, \beta \geq 0$ with $\alpha+\beta=1$.

For modular $\rho$ on $X$, the space

$$
X_{\rho}=\left\{x \in X: \rho(\lambda x) \longrightarrow 0 \text { as } \lambda \longrightarrow 0^{+}\right\}
$$

is called the modular space.

A sequence $\left(x_{n}\right)$ in $X_{\rho}$ is called modular convergent to $x \in X_{\rho}$ if there exists a $\lambda>0$ such that $\rho\left(\lambda\left(x_{n}-x\right)\right) \rightarrow 0$ as $n \rightarrow \infty$.

A modular $\rho$ is said to satisfy the $\Delta_{2}$-condition $\left(\rho \in \Delta_{2}\right.$ ) if for any $\varepsilon>0$ there exist constants $K \geq 2$ and $a>0$ such that

$$
\rho(2 u) \leq K \rho(u)+\varepsilon
$$

for all $u \in X_{\rho}$ with $\rho(u) \leq a$.

If $\rho$ satisfies the $\Delta_{2}$-condition for any $a>0$ with $K \geq 2$ dependent on $a$, we say that $\rho$ satisfies the strong $\Delta_{2}$-condition $\left(\rho \in \Delta_{2}^{S}\right)$.

By a lacunary sequence $\theta=\left(k_{r}\right)$, where $k_{0}=0$, we will mean an increasing sequence of nonnegative integers with $k_{r}-k_{r-1} \rightarrow \infty$ as $r \rightarrow \infty$. The intervals determined by $\theta$ will be denote by $I_{r}=\left(k_{r-1}, k_{r}\right]$. We write $h_{r}=k_{r}-k_{r-1}$ and the ratio $k_{r} / k_{r-1}$, will be denoted by $q_{r}$. The space of lacunary strongly convergent sequence $N_{\theta}$ was defined by Freedman et al. [19] as

$$
N_{\theta}=\left\{x=\left(x_{k}\right): \lim _{r \rightarrow \infty} \frac{1}{h_{r}} \sum_{k \in I_{r}}\left|x_{k}-l\right|=0, \text { for some } l\right\} .
$$

It is well known that there is very closed connection between the space of lacunary strongly convergent sequence and the space of strongly Cesaro summability sequences. This connection can be found in [18-23], because a lot of these connection, a lot of geometric property of Cesaro sequence spaces can generalize the lacunary sequence spaces.

Let $w$ be the space of all real sequences. Let $p=\left(p_{r}\right)$ be a bounded sequence of the positive real numbers. In 2007, Karakaya [24] introduced the new sequence spaces $l(p, \theta)$ involving lacunary sequence as follows:

$$
l(p, \theta)=\left\{x=(x(i)): \sum_{r=1}^{\infty}\left(\frac{1}{h_{r}} \sum_{i \in I_{r}}|x(i)|\right)^{p_{r}}<\infty\right\}
$$


and paranorm on $l(p, \theta)$ is given by

$$
\|x\|_{l(p, \theta)}=\left(\sum_{r=1}^{\infty}\left(\frac{1}{h_{r}} \sum_{i \in I_{r}}|x(i)|\right)^{p_{r}}\right)^{1 / M}
$$

where $M=\sup _{r} p_{r}$. If $p_{r}=p$ for all $r \in \mathbb{N}$, we will use the notation $l_{p}(\theta)$ in place of $l(p, \theta)$. The norm on $l_{p}(\theta)$ is given by

$$
\|x\|_{l_{p}(\theta)}=\left(\sum_{r=1}^{\infty}\left(\frac{1}{h_{r}} \sum_{i \in I_{r}}|x(i)|\right)^{p}\right)^{1 / p} .
$$

By using the properties of lacunary sequence in the space $l(p, \theta)$, we get the following sequences. If $\theta=\left(2^{r}\right)$, then $l(p, \theta)=\operatorname{ces}(p)$. If $\theta=\left(2^{r}\right)$ and $p_{r}=p$ for all $r \in \mathbb{N}$, then $l(p, \theta)=\operatorname{ces}_{p}$. For $x \in l(p, \theta)$ defined the modular on $l(p, \theta)$ by

$$
\rho(x)=\sum_{r=1}^{\infty}\left(\frac{1}{h_{r}} \sum_{i \in I_{r}}|x(i)|\right)^{p_{r}} .
$$

It is easy to see that if $\lim _{r \rightarrow \infty} \sup p_{r}<\infty$ then $\rho \in \Delta_{2}^{s}$. The Luxembourg norm on $l(p, \theta)$ is defined by

$$
\|x\|=\inf \left\{\varepsilon>0: \rho\left(\frac{x}{\varepsilon}\right) \leq 1\right\} .
$$

The Luxembourg norm on $l_{p}(\theta)$ can be reduced to a usual norm on $l_{p}(\theta)[24]$, that is,

$$
\|x\|=\|x\|_{l_{p}(\theta)} .
$$

Throughout this paper, we assume that $\lim _{r \rightarrow \infty} \inf p_{r}>1$ and $\lim _{r \rightarrow \infty} \sup p_{r}<\infty$ and for $x \in w, i \in \mathbb{N}$, we denote

$$
\begin{gathered}
e_{i}=(\overbrace{0,0, \ldots, 0}^{i-1 \text { times }}, 1,0,0,0, \ldots), \\
\left.x\right|_{i}=(x(1), x(2), x(3), \ldots, x(i), 0,0,0, \ldots), \\
\left.x\right|_{\mathbb{N}-i}=(0,0,0, \ldots, x(i+1), x(i+2), \ldots) .
\end{gathered}
$$

The following results are very important for our consideration. 
Lemma 1.1 (see [25, Lemma 2.1]). If $\rho \in \Delta_{2}^{s}$, then for any $L>0$ and $\varepsilon>0$, there exists $\delta=$ $\delta(L, \varepsilon)>0$ such that

$$
|\rho(u+v)-\rho(u)|<\varepsilon,
$$

whenever $u, v \in X_{\rho}$ with $\rho(u) \leq L$, and $\rho(v) \leq \delta$.

Lemma 1.2 (see [25, Lemma 2.3]). Convergence in norm and in modular are equivalent in $X_{\rho}$ if $\rho \in \Delta_{2}$.

Lemma 1.3 (see [25, Corollary 2.2, Lemma 2.3]). If $\rho \in \Delta_{2}$, then for any sequence $\left(x_{n}\right)$ in $X_{\rho}$, $\left\|x_{n}\right\| \rightarrow 0$ if and only if $\rho\left(x_{n}\right) \rightarrow 0$ as $n \rightarrow \infty$.

Lemma 1.4 (see [25, Lemma 2.4]). If $\rho \in \Delta_{2}^{s}$, then for any $\varepsilon>0$ there exists $\delta=\delta(\varepsilon)>0$ such that $\|x\| \geq 1+\delta$ whenever $\rho(x) \geq 1+\varepsilon$.

Lemma 1.5 (see [24, Lemma 2.3]). The functional $\rho$ is a convex modular on $l(p, \theta)$.

Lemma 1.6 (see [24, Lemma 2.5]). (i) For any $x \in l(p, \theta)$, if $\|x\|<1$, then $\rho(x) \leq\|x\|$.

(ii) For any $x \in l(p, \theta),\|x\|=1$ if and only if $\rho(x)=1$.

\section{The Main Results}

In this section, we prove the property $(\beta)$ and uniform Opial property in lacunary sequence and connect to the fixed point property. First we shall give some results which are very important for our consideration.

Lemma 2.1. For any $x \in l(p, \theta)$, there exists $k_{0} \in \mathbb{N}$ and $\lambda \in(0,1)$ such that $\varphi\left(x^{k} / 2\right) \leq((1-$ l)/2) $Q\left(x^{k}\right)$ for all $k \in \mathbb{N}$ with $k \geq k_{0}$, where

$$
x^{k}=(\overbrace{0,0, \ldots, 0}^{k-1}, x(k), x(k+1), x(k+2), \ldots) .
$$

Proof. Let $k \in \mathbb{N}$ be fixed. So there exist $r_{k} \in \mathbb{N}$ such that $k \in I_{r_{k}}$. Let $\alpha$ be a real number such that $1<\alpha \leq \lim _{r \rightarrow \infty} \inf p_{r}$, then there exists $k_{0} \in \mathbb{N}$ such that $\alpha<p_{r_{k}}$ for all $k \geq k_{0}$. Choose $\lambda \in(0,1)$ as a real such that $(1 / 2)^{\alpha} \leq(1-\lambda) / 2$. Then for each $x \in l(p, \theta)$ and $k \geq k_{0}$, we have

$$
\begin{aligned}
\varrho\left(\frac{x^{k}}{2}\right) & =\sum_{r=r_{k}}^{\infty}\left(\frac{1}{h_{r}} \sum_{i \in I_{r}}\left|\frac{x(i)}{2}\right|\right)^{p_{r}} \\
& =\sum_{r=r_{k}}^{\infty}\left(\frac{1}{2}\right)^{p_{r}}\left(\frac{1}{h_{r}} \sum_{i \in I_{r}}|x(i)|\right)^{p_{r}}
\end{aligned}
$$




$$
\begin{aligned}
& \leq\left(\frac{1}{2}\right)^{\alpha} \sum_{r=r_{k}}^{\infty}\left(\frac{1}{h_{r}} \sum_{i \in I_{r}}|x(i)|\right)^{p_{r}} \\
& \leq \frac{1-\lambda}{2} \rho\left(x^{k}\right) .
\end{aligned}
$$

Lemma 2.2. For any $x \in l(p, \theta)$ and $\varepsilon \in(0,1)$ there exists $\delta \in(0,1)$ such that $Q(x) \leq 1-\varepsilon$ implies $\|x\| \leq 1-\delta$.

Proof. Suppose that the lemma does not hold, then there exist $\varepsilon>0$ and $x_{n} \in l(p, \theta)$ such that $\rho\left(x_{n}\right) \leq 1-\varepsilon$ and $1 / 2 \leq\left\|x_{n}\right\| \nearrow 1$. Let $a_{n}=\left(1 /\left\|x_{n}\right\|\right)-1$. Then $a_{n} \rightarrow 0$ as $n \rightarrow \infty$. Let $L=\sup \left\{\rho\left(2 x_{n}\right): n \in \mathbb{N}\right\}$. Since $\rho \in \Delta_{2}^{s}$ there exists $K \geq 2$ such that

$$
\rho(2 u) \leq K \rho(u)+1,
$$

for every $u \in l(p, \theta)$ with $\rho(u)<1$. By (2.3), we have $\rho\left(2 x_{n}\right) \leq K \rho\left(x_{n}\right)+1 \leq K+1$ for all $n \in \mathbb{N}$. Hence $0<L<\infty$. By Lemmas 1.5 and 1.6(ii), we have

$$
\begin{aligned}
1=\rho\left(\frac{x_{n}}{\left\|x_{n}\right\|}\right) & =\rho\left(2 a_{n} x_{n}+\left(1-a_{n}\right) x_{n}\right) \\
& \leq a_{n} \rho\left(2 x_{n}\right)+\left(1-a_{n}\right) \rho\left(x_{n}\right) \\
& \leq a_{n} L+(1-\varepsilon) \longrightarrow 1-\varepsilon,
\end{aligned}
$$

which is a contradiction.

Theorem 2.3. The space $l(p, \theta)$ is Banach spaces with respect to the Luxemburg norm.

Proof. Let $\left(x_{n}\right)=\left(x_{n}(i)\right)$ be a Cauchy sequence in $l(p, \theta)$ and $\varepsilon \in(0,1)$. Thus there exists $N \in \mathbb{N}$ such that $\left\|x_{n}-x_{m}\right\|<\varepsilon^{M}$ for all $n, m \geq N$. By Lemma 1.6 (i), we have

$$
\rho\left(x_{n}-x_{m}\right) \leq\left\|x_{n}-x_{m}\right\|<\varepsilon^{M} \quad \forall n, m \geq N .
$$

That is,

$$
\sum_{r=1}^{\infty}\left(\frac{1}{h_{r}} \sum_{i \in I_{r}}\left|x_{n}(i)-x_{m}(i)\right|\right)^{p_{r}}<\varepsilon^{M} \quad \forall n, m \geq N .
$$

For fixed $r$, we get that

$$
\left|x_{n}(i)-x_{m}(i)\right|<\varepsilon \quad \forall n, m \geq N .
$$


Thus let $\left(x_{n}(i)\right)$ be a Cauchy sequence in $\mathbb{R}$ for all $i \in \mathbb{N}$. Since $\mathbb{R}$ is complete, then there exists $x(i) \in \mathbb{R}$ such that $x_{m}(i) \rightarrow x(i)$ as $m \rightarrow \infty$ for all $i \in \mathbb{N}$. Thus for fixed $r$, we have

$$
\left|x_{n}(i)-x(i)\right|<\varepsilon \quad \text { as } m \longrightarrow \infty, \forall n \geq N
$$

This implies that, for all $n \geq N$,

$$
\rho\left(x_{n}-x_{m}\right) \longrightarrow \rho\left(x_{n}-x\right) \text { as } m \longrightarrow \infty
$$

This means that, for all $n \geq N$,

$$
\sum_{r=1}^{\infty}\left(\frac{1}{h_{r}} \sum_{i \in I_{r}}\left|x_{n}(i)-x_{m}(i)\right|\right)^{p_{r}} \longrightarrow \sum_{r=1}^{\infty}\left(\frac{1}{h_{r}} \sum_{i \in I_{r}}\left|x_{n}(i)-x(i)\right|\right)^{p_{r}}
$$

as $m \rightarrow \infty$. By (2.6), we have

$$
\varrho\left(x_{n}-x\right) \leq\left\|x_{n}-x_{m}\right\|<\varepsilon^{M} \leq \varepsilon \quad \forall n \geq N .
$$

This implies that $x_{n} \rightarrow x$ as $n \rightarrow \infty$. So we have $x_{N}-x \in l(p, \theta)$. By the linearity of the sequence space $l(p, \theta)$, we have $x=\left(x_{N}-x\right)+x_{N} \in l(p, \theta)$. Therefore the sequence space $l(p, \theta)$ is Banach space, with respect to the Luxemburg norm and the proof is complete.

Theorem 2.4. The space $l(p, \theta)$ has property $(\beta)$.

Proof. Let $\varepsilon>0$ and $\left(x_{n}\right) \subset B(l(p, \theta))$ with $\operatorname{sep}\left(x_{n}\right) \geq \varepsilon$. For each $k \in \mathbb{N}$, there exist $r_{k} \in \mathbb{N}$ such that $k$ is a minimal element in $I_{r_{k}}$. Let

$$
x_{n}^{k}=(\overbrace{0,0, \ldots, 0}^{k-1}, x_{n}(k), x_{n}(k+1), x_{n}(k+2), \ldots) .
$$

Since for each $i \in \mathbb{N},\left(x_{n}(i)\right)_{n=1}^{\infty}$ is bounded. By using the diagonal method, we have that for each $k \in \mathbb{N}$ we can find subsequence $\left(x_{n_{j}}\right)$ of $\left(x_{n}\right)$ such that $\left(x_{n_{j}}(i)\right)$ converges for each $i \in \mathbb{N}$. Therefore, for any $k \in \mathbb{N}$ there exists an increasing sequence $\left(t_{k}\right)$ such that $\operatorname{sep}\left(\left(x_{n_{j}}^{k}\right)_{j>t_{k}}\right) \geq \varepsilon$. Hence for each $k \in \mathbb{N}$ there exists sequence of positive integers $\left(s_{k}\right)_{k=1}^{\infty}$ with $s_{1}<s_{2}<s_{3}<\cdots$ such that $\left\|x_{s_{k}}^{k}\right\| \geq \varepsilon / 2$, and since $\rho \in \Delta_{2}^{s}$, by Lemma 1.3 we may assume that there exists $\eta>0$ such that $\rho\left(x_{s_{k}}^{k}\right) \geq \eta$ for all $k \in \mathbb{N}$, that is,

$$
\sum_{r=r_{k}}^{\infty}\left(\frac{1}{h_{r}} \sum_{i \in I_{r}}\left|x_{s_{k}}^{k}(i)\right|\right)^{p_{r}} \geq \eta
$$


for all $k \in \mathbb{N}$. On the other hand by Lemma 2.1, there exist $k_{0} \in \mathbb{N}$ and $\lambda \in(0,1)$ such that

$$
\rho\left(\frac{u^{k}}{2}\right) \leq \frac{1-\lambda}{2} \rho\left(u^{k}\right)
$$

for all $u \in l(p, \theta)$ and $k \geq k_{0}$. From Lemma 2.2, there exist $\delta \in(0,1)$ such that for any $y \in$ $l(p, \theta)$,

$$
\rho(y) \leq 1-\frac{\lambda \eta}{4} \Longrightarrow\|y\| \leq 1-\delta
$$

Since again $\varrho \in \Delta_{2}^{s}$, by Lemma 1.1, there exists $\delta_{0}$ such that

$$
|\rho(u+v)-\rho(u)|<\frac{\lambda \eta}{4}
$$

whenever $\rho(u) \leq 1$ and $\rho(v) \leq \delta_{0}$. Since $x \in B(l(p, \theta))$, we have that $\rho(x) \leq 1$. Then there exits $k \geq k_{0}$ such that $\rho\left(x^{k}\right) \leq \delta_{0}$. We put $u=x_{s_{k}}^{k}$ and $v=x^{k}$,

$$
\rho\left(\frac{u}{2}\right)=\sum_{r=r_{k}}^{\infty}\left(\frac{1}{h_{r}} \sum_{i \in I_{r}}\left|\frac{x_{s_{k}}(i)}{2}\right|\right)^{p_{r}}<1, \quad \rho\left(\frac{v}{2}\right)=\sum_{r=r_{k}}^{\infty}\left(\frac{1}{h_{r}} \sum_{i \in I_{r}}\left|\frac{x(i)}{2}\right|\right)^{p_{r}}<\delta_{0} .
$$

From (2.14) and (2.16), we have

$$
\sum_{r=r_{k}}^{\infty}\left(\frac{1}{h_{r}} \sum_{i \in I_{r}}\left|\frac{x(i)+x_{s_{k}}(i)}{2}\right|\right)^{p_{r}}=\rho\left(\frac{u+v}{2}\right) \leq \rho\left(\frac{u}{2}\right)+\frac{\lambda \eta}{4} \leq \frac{1-\lambda}{2}(\rho(u))+\frac{\lambda \eta}{4} .
$$

By (2.13), (2.16), (2.18), and convexity of function $f(t)=|t|^{p_{r}}$, for all $r \in \mathbb{N}$, we have

$$
\begin{aligned}
\varrho\left(\frac{x+x_{s_{k}}}{2}\right) & =\sum_{r=1}^{\infty}\left(\frac{1}{h_{r}} \sum_{i \in I_{r}}\left|\frac{x(i)+x_{s_{k}}(i)}{2}\right|\right)^{p_{r}} \\
& =\sum_{r=1}^{r_{k}-1}\left(\frac{1}{h_{r}} \sum_{i \in I_{r}}\left|\frac{x(i)+x_{s_{k}}(i)}{2}\right|\right)^{p_{r}}+\sum_{r=r_{k}}^{\infty}\left(\frac{1}{h_{r}} \sum_{i \in I_{r}}\left|\frac{x(i)+x_{s_{k}}(i)}{2}\right|\right)^{p_{r}} \\
& \leq \frac{1}{2}\left(\sum_{r=1}^{r_{k}-1}\left(\frac{1}{h_{r}} \sum_{i \in I_{r}}|x(i)|\right)^{p_{r}}+\sum_{r=1}^{r_{k}-1}\left(\frac{1}{h_{r}} \sum_{i \in I_{r}}\left|x_{S_{k}}(i)\right|\right)^{p_{r}}\right)
\end{aligned}
$$


Abstract and Applied Analysis

$$
\begin{aligned}
& +\sum_{r=r_{k}}^{\infty}\left(\frac{1}{h_{r}} \sum_{i \in I_{r}}\left|\frac{x_{s_{k}}(i)}{2}\right|\right)^{p_{r}}+\frac{\lambda \eta}{4} \\
\leq & \frac{1}{2}\left(\sum_{r=1}^{r_{k}-1}\left(\frac{1}{h_{r}} \sum_{i \in I_{r}}|x(i)|\right)^{p_{r}}+\sum_{r=1}^{r_{k}-1}\left(\frac{1}{h_{r}} \sum_{i \in I_{r}}\left|x_{s_{k}}(i)\right|\right)^{p_{r}}\right) \\
& +\frac{1-\lambda}{2} \sum_{r=r_{k}}^{\infty}\left(\frac{1}{h_{r}} \sum_{i \in I_{r}}\left|x_{s_{k}}(i)\right|\right)^{p_{r}}+\frac{\lambda \eta}{4} \\
= & \frac{1}{2} \sum_{r=1}^{r_{k}-1}\left(\frac{1}{h_{r}} \sum_{i \in I_{r}}|x(i)|\right)^{p_{r}}+\frac{1}{2} \sum_{r=1}^{r_{k}-1}\left(\frac{1}{h_{r}} \sum_{i \in I_{r}}\left|x_{s_{k}}(i)\right|\right)^{p_{r}} \\
& +\frac{1-\lambda}{2} \sum_{r=r_{k}}^{\infty}\left(\frac{1}{h_{r}} \sum_{i \in I_{r}}\left|x_{s_{k}}(i)\right|\right)^{p_{r}}+\frac{\lambda \eta}{4} \\
= & \frac{1}{2} \sum_{r=1}^{r_{k}-1}\left(\frac{1}{h_{r}} \sum_{i \in I_{r}}|x(i)|\right)^{p_{r}}+\frac{1}{2} \sum_{r=1}^{\infty}\left(\frac{1}{h_{r}} \sum_{i \in I_{r}}\left|x_{s_{k}}(i)\right|\right)^{p_{r}} \\
& -\frac{\lambda}{2} \sum_{r=r_{k}}^{\infty}\left(\frac{1}{h_{r}} \sum_{i \in I_{r}}\left|x_{s_{k}}(i)\right|\right)^{p_{r}}+\frac{\lambda \eta}{4} \\
\leq & \frac{1}{2}+\frac{1}{2}-\frac{\lambda \eta}{2}+\frac{\lambda \eta}{4} \\
= & 1-\frac{\lambda \eta}{4} .
\end{aligned}
$$

So it follows from (2.15) that

$$
\left\|\frac{x+x_{s_{k}}}{2}\right\| \leq 1-\delta
$$

Therefore, the space $l(p, \theta)$ has property $(\beta)$.

\section{Theorem 2.4.}

By the facts presented in the introduction, following results are obtained directly from

Corollary 2.5. The space $l_{p}(\theta)$ has property $(\beta)$.

Corollary 2.6. The space $l(p, \theta)$ is nearly uniform convexity, has drop property, and is reflexive.

Corollary 2.7. The space $l(p, \theta)$ has property (UKK).

Corollary 2.8 (see [24, Theorem 2.9]). The space $l(p, \theta)$ has property $(H)$.

Corollary 2.9. The space $l_{p}(\theta)$ is nearly uniform convexity, has drop property, and is reflexive. 
Corollary 2.10. The space $l_{p}(\theta)$ has property $(U K K)$ and $(H)$.

Theorem 2.11. The space $l(p, \theta)$ has uniform Opial property.

Proof. Take any $\varepsilon>0$ and $x \in l(p, \theta)$ with $\|x\| \geq \varepsilon$. Let $\left(x_{n}\right)$ be weakly null sequence in $S(l(p, \theta))$. By $\lim _{r \rightarrow \infty} \sup p_{r}<\infty$, that is, $\rho \in \Delta_{2}^{s}$, hence by Lemma 1.2 there exists $\delta \in(0,1)$ independent of $x$ such that $\rho(x)>\delta$. Also, by $\rho \in \Delta_{2}^{s}$, Lemma 1.1 asserts that there exists $\delta_{1} \in(0, \delta)$ such that

$$
|\rho(y+z)-\rho(y)|<\frac{\delta}{4}
$$

whenever $\rho(y) \leq 1$ and $\rho(z) \leq \delta_{1}$. Choose $r_{0} \in \mathbb{N}$ such that

$$
\sum_{r=r_{0}+1}^{\infty}\left(\frac{1}{h_{r}} \sum_{i \in I_{r}}|x(i)|\right)^{p_{r}}<\frac{\delta_{1}}{4} .
$$

So, we have

$$
\begin{aligned}
\delta & <\sum_{r=1}^{r_{0}}\left(\frac{1}{h_{r}} \sum_{i \in I_{r}}|x(i)|\right)^{p_{r}}+\sum_{r=r_{0}+1}^{\infty}\left(\frac{1}{h_{r}} \sum_{i \in I_{r}}|x(i)|\right)^{p_{r}} \\
& \leq \sum_{r=1}^{r_{0}}\left(\frac{1}{h_{r}} \sum_{i \in I_{r}}|x(i)|\right)^{p_{r}}+\frac{\delta_{1}}{4},
\end{aligned}
$$

which implies that

$$
\sum_{r=1}^{r_{0}}\left(\frac{1}{h_{r}} \sum_{i \in I_{r}}|x(i)|\right)^{p_{r}}>\delta-\frac{\delta_{1}}{4}>\delta-\frac{\delta}{4}=\frac{3 \delta}{4} .
$$

Since $x_{n} \stackrel{w}{\rightarrow} 0$, then there exists $n_{0} \in \mathbb{N}$ such that

$$
\frac{3 \delta}{4} \leq \sum_{r=1}^{r_{0}}\left(\frac{1}{h_{r}} \sum_{i \in I_{r}}\left|x_{n}(i)+x(i)\right|\right)^{p_{r}}
$$

for all $n>n_{0}$, since weak convergence implies coordinatewise convergence. Again, by $x_{n} \stackrel{w}{\longrightarrow}$ 0 , then there exists $n_{1} \in \mathbb{N}$ such that

$$
\left\|x_{\left.n\right|_{k_{0}}}\right\|<1-\left(1-\frac{\delta}{4}\right)^{1 / M}
$$


for all $n>n_{1}$ where $k_{0}$ is a minimal element in $I_{r_{0}+1}$ and $M \in \mathbb{N}$ with $p_{r} \leq M$ for all $r \in \mathbb{N}$. Hence, by the triangle inequality of the norm, we get

$$
\left\|x_{\left.n\right|_{\mathbb{N}-k_{0}}}\right\|>\left(1-\frac{\delta}{4}\right)^{1 / M}
$$

It follows by the definition of $\|\cdot\|$ that we have

$$
\begin{aligned}
1 & <\varphi\left(\frac{x_{\left.n\right|_{\mathbb{N}-k_{0}}}}{(1-(\delta / 4))^{1 / M}}\right) \\
& =\sum_{r=r_{0}+1}^{\infty}\left(\frac{\left(1 / h_{r}\right) \sum_{i \in I_{r}}\left|x_{n}(i)\right|}{(1-(\delta / 4))^{1 / M}}\right)^{p_{r}} \\
& \leq\left(\frac{1}{(1-(\delta / 4))^{1 / M}}\right)^{M} \sum_{r=r_{0}+1}^{\infty}\left(\frac{1}{h_{r}} \sum_{i \in I_{r}}\left|x_{n}(i)\right|\right)^{p_{r}}
\end{aligned}
$$

Which implies that

$$
\sum_{r=r_{0}+1}^{\infty}\left(\frac{1}{h_{r}} \sum_{i \in I_{r}}\left|x_{n}(i)\right|\right)^{p_{r}}>1-\frac{\delta}{4}
$$

for all $n>n_{1}$. By inequality (2.21), (2.25), and (2.29), it yields for any $n>n_{1}$ that

$$
\begin{aligned}
\varrho\left(x_{n}+x\right) & =\sum_{r=1}^{r_{0}}\left(\frac{1}{h_{r}} \sum_{i \in I_{r}}\left|x_{n}(i)+x(i)\right|\right)^{p_{r}}+\sum_{r=r_{0}+1}^{\infty}\left(\frac{1}{h_{r}} \sum_{i \in I_{r}}\left|x_{n}(i)+x(i)\right|\right)^{p_{r}} \\
& \geq \frac{3 \delta}{4}+\sum_{r=r_{0}+1}^{\infty}\left(\frac{1}{h_{r}} \sum_{i \in I_{r}}\left|x_{n}(i)\right|\right)^{p_{r}}-\frac{\delta}{4} \\
& \geq \frac{3 \delta}{4}+\left(1-\frac{\delta}{4}\right)-\frac{\delta}{4} \\
& \geq 1+\frac{\delta}{4} .
\end{aligned}
$$

Since $\rho \in \Delta_{2}^{s}$ and by Lemma 1.4 there exists $\tau$ depending on $\delta$ only such that $\left\|x_{n}+x\right\| \geq 1+\tau$, which implies that $\lim _{n \rightarrow \infty} \inf \left\|x_{n}+x\right\| \geq 1+\tau$, hence the prove is complete.

By the facts presented in the introduction and the reflexivity of $l(p, \theta)$, we get the following results.

Corollary 2.12. The space $l_{p}(\theta)$ has uniform Opial property.

Corollary 2.13. The space $l(p, \theta)$ has property $(L)$ and the fixed point property.

Corollary 2.14. The space $l_{p}(\theta)$ has property $(L)$ and the fixed point property. 


\section{Acknowledgments}

The authors are grateful to the referees for their valuable comments. They also wish to thank the Higher Education Research Promotion and National Research University Project of Thailand, Office of the Higher Education Commission for financial support (under the Project no. 54000267). Mr. Chirasak Mongkolkeha was supported by the Thailand Research Fund through the Royal Golden Jubilee Program under Grant PHD/0029/2553 for Ph.D. program at KMUTT, Thailand. The second author was supported by the Commission on Higher Education, the Thailand Research Fund, and the King Mongkuts University of Technology Thonburi (Grant no. MRG5380044).

\section{References}

[1] J. A. Clarkson, "Uniformly convex spaces," Transactions of the American Mathematical Society, vol. 40, no. 3, pp. 396-414, 1936.

[2] D. Kutzarova, "An isomorphic characterization of property $(\beta)$ of Rolewicz," Note di Matematica, vol. 10, no. 2, pp. 347-354, 1990.

[3] R. Huff, "Banach spaces which are nearly uniformly convex," The Rocky Mountain Journal of Mathematics, vol. 10, no. 4, pp. 743-749, 1980.

[4] S. Chen, Dissertationes Mathematicae(Rozprawy Matematyczne), Instytut Matematyczny PAN, Warszawa, Poland, 1996.

[5] V. Montesinos, "Drop property equals reflexivity," Studia Mathematica, vol. 87, no. 1, pp. 93-100, 1987.

[6] S. Rolewicz, "On $\Delta$-uniform convexity and drop property," Studia Mathematica, vol. 87, no. 2, pp. 181-191, 1987.

[7] Z. Opial, "Weak convergence of the sequence of successive approximations for nonexpansive mappings," Bulletin of the American Mathematical Society, vol. 73, pp. 591-597, 1967.

[8] S. Prus, "Banach spaces with the uniform Opial property," Nonlinear Analysis, Theory, Methods $\mathcal{E}$ Applications, vol. 18, no. 8, pp. 697-704, 1992.

[9] Y. Cui and H. Hudzik, "Some geometric properties related to fixed point theory in Cesàro spaces," Collectanea Mathematica, vol. 50, no. 3, pp. 277-288, 1999.

[10] N. Petrot and S. Suantai, "Uniform Opial properties in generalized Cesàro sequence spaces," Nonlinear Analysis, Theory, Methods \& Applications, vol. 63, no. 8, pp. 1116-1125, 2005.

[11] J.-P. Gossez and E. Lami Dozo, "Some geometric properties related to the fixed point theory for nonexpansive mappings," Pacific Journal of Mathematics, vol. 40, pp. 565-573, 1972.

[12] S. Dhompongsa and A. Kaewkhao, "A note on properties that imply the fixed point property," Abstract and Applied Analysis, vol. 2006, Article ID 34959, 12 pages, 2006.

[13] M. A. Japón, "Some geometric properties in modular spaces and application to fixed point theory," Journal of Mathematical Analysis and Applications, vol. 295, no. 2, pp. 576-594, 2004.

[14] H. B. Thompson and Y. Cui, "The fixed point property in Musielak-Orlicz sequence spaces," Commentationes Mathematicae Universitatis Carolinae, vol. 42, no. 2, pp. 299-309, 2001.

[15] K. Kuratowski, "Sur les espaces complets," Fundamenta Mathematicae, vol. 15, pp. 301-309, 1930.

[16] K. Goebel and W. A. Kirk, Topics in Metric Fixed Point Theory, vol. 28 of Cambridge Studies in Advanced Mathematics, Cambridge University Press, Cambridge, UK, 1990.

[17] W. A. Kirk, "A fixed point theorem for mappings which do not increase distances," The American Mathematical Monthly, vol. 72, pp. 1004-1006, 1965.

[18] K. Deimling, Nonlinear Functional Analysis, Springer, Berlin, Germany, 1985.

[19] A. R. Freedman, J. J. Sember, and M. Raphael, "Some Cesàro-type summability spaces," Proceedings of the London Mathematical Society, vol. 37, no. 3, pp. 508-520, 1978.

[20] J. A. Fridy and C. Orhan, "Lacunary statistical summability," Journal of Mathematical Analysis and Applications, vol. 173, no. 2, pp. 497-504, 1993.

[21] V. Karakaya, "On lacunary $\sigma$-statistical convergence," Information Sciences. An International Journal, vol. 166, no. 1-4, pp. 271-280, 2004.

[22] M. Mursaleen and T. A. Chishti, "Some spaces of lacunary sequences defined by the modulus," Journal of Analysis, vol. 4, pp. 153-159, 1996.

[23] S. Pehlivan and B. Fisher, "Lacunary strong convergence with respect to a sequence of modulus functions," Commentationes Mathematicae Universitatis Carolinae, vol. 36, no. 1, pp. 69-76, 1995. 
[24] V. Karakaya, "Some geometric properties of sequence spaces involving lacunary sequence," Journal of Inequalities and Applications, vol. 2007, Article ID 81028, 8 pages, 2007.

[25] Y. Cui and H. Hudzik, "On the uniform Opial property in some modular sequence spaces," Functiones et Approximatio Commentarii Mathematici, vol. 26, pp. 93-102, 1998. 


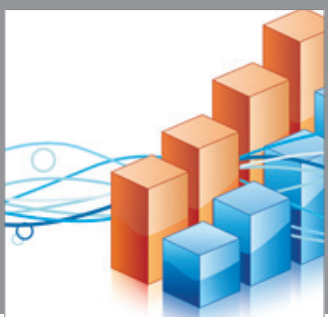

Advances in

Operations Research

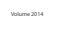

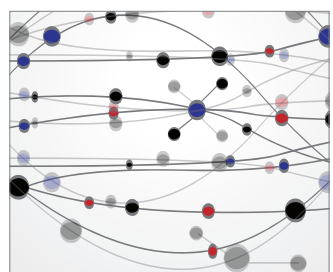

\section{The Scientific} World Journal
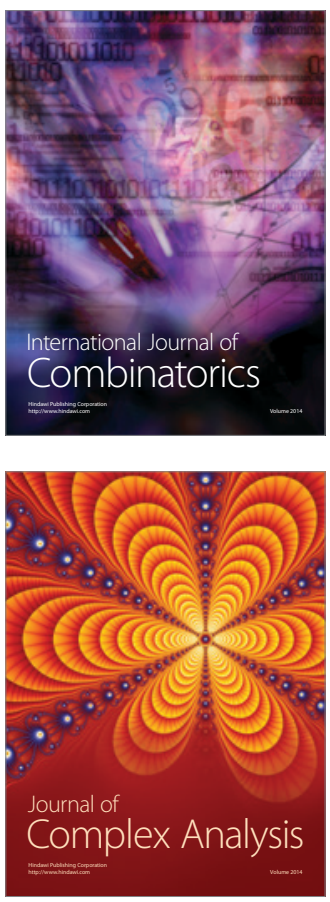

International Journal of

Mathematics and

Mathematical

Sciences
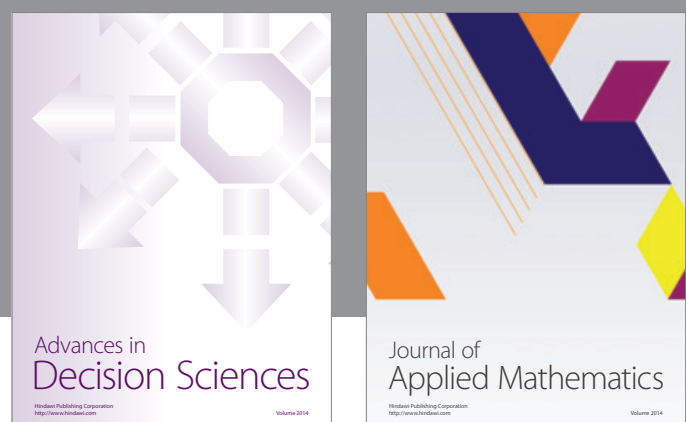

Journal of

Applied Mathematics
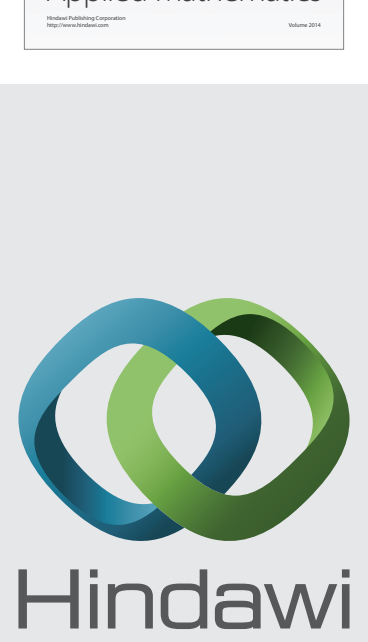

Submit your manuscripts at http://www.hindawi.com
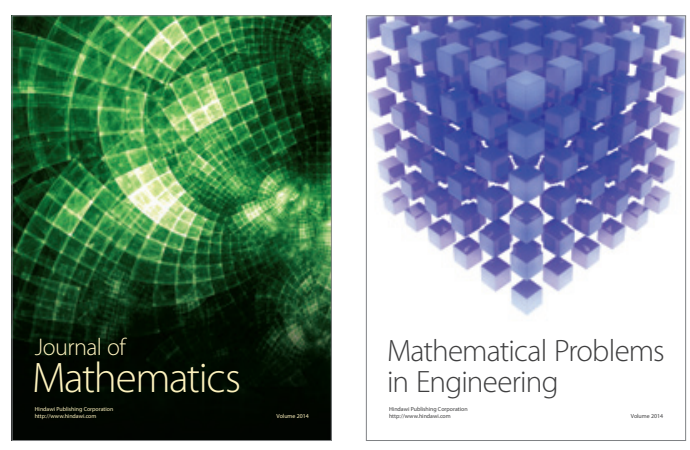

Mathematical Problems in Engineering
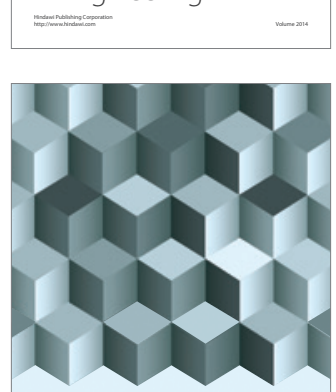

Journal of

Function Spaces
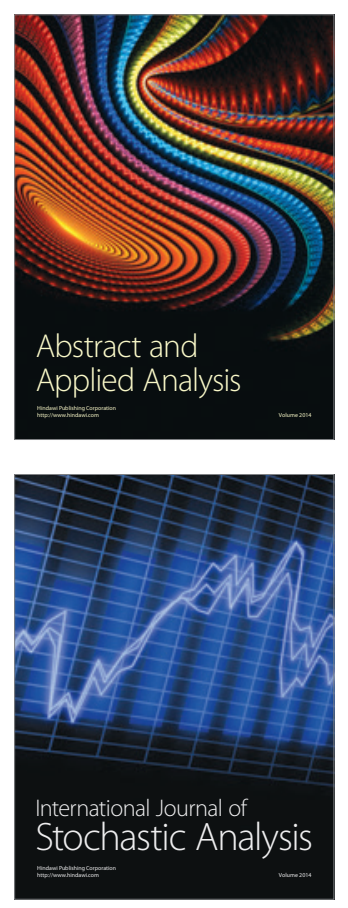

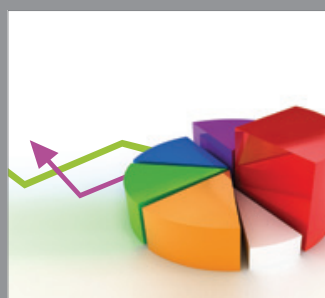

ournal of

Probability and Statistics

Promensencen
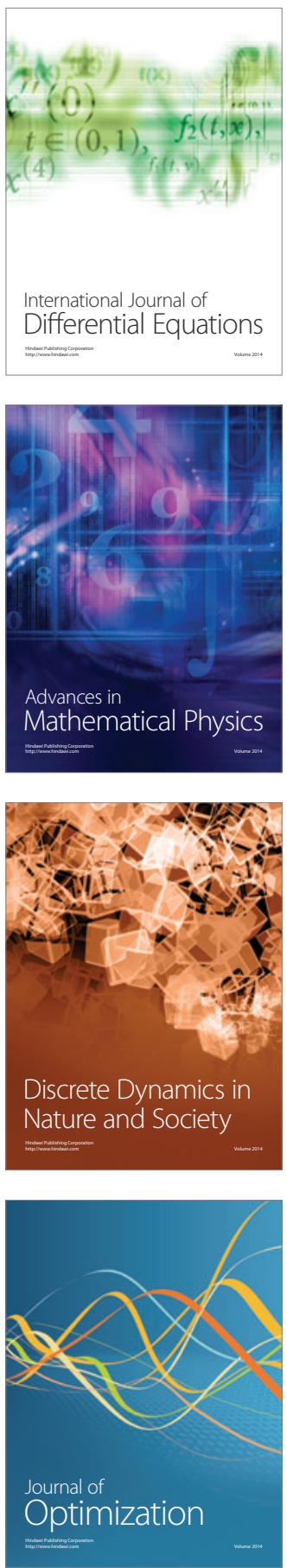